\title{
Candidate Stability and Nonbinary Social ChOICE
}

by

John A. Weymark

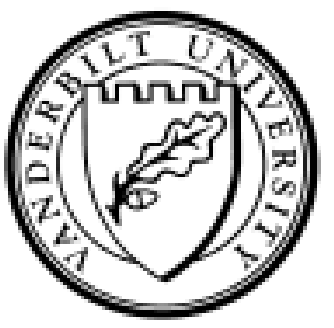

Working Paper No. 00-W29R

July 2000

Revised February 2001

DEPARTMENT OF ECONOMICS

VANDERBILT UNIVERSITY

NASHVILLE, TN 37235

www.vanderbilt.edu/econ 


\title{
Candidate Stability and Nonbinary Social Choice*
}

\author{
John A. Weymark \\ Department of Economics, Vanderbilt University, \\ Box 1819, Station B, Nashville, TN 37235 \\ (e-mail: john.weymark@vanderbilt.edu)
}

July 2000

Revised, February 2001

\begin{abstract}
A voting procedure is candidate stable if no candidate would prefer to withdraw from an election when all of the other potential candidates enter. Dutta, Jackson, and Le Breton have recently established a number of theorems showing that candidate stability is incompatible with some other desirable properties of voting procedures. This article shows that Grether and Plott's nonbinary generalization of Arrow's Theorem can be used to provide a simple proof of Dutta, Jackson, and Le Breton's impossibility theorem for the case in which the voters and potential candidates have no one in common.

Journal of Economic Literature Classification Numbers: D71, D72.
\end{abstract}

*I am grateful to Michel Le Breton for his comments and to Lars Ehlers for a suggestion that led to a simplification of the proof of Theorem 2. 


\section{INTRODUCTION}

A voting procedure is candidate stable if no candidate would prefer to withdraw from an election when all of the other potential candidates enter. In other words, it is a Nash equilibrium for all potential candidates to stand for election. Dutta, Jackson, and Le Breton [2] have recently shown that candidate stability is incompatible with some other desirable properties of voting procedures. They have established impossibility theorems both for the case in which there is no overlap between the candidates and voters and for the case in which there is. In this article, I show that Grether and Plott's [3] nonbinary generalization of Arrow's [1] Theorem can be used to provide a simple proof of Dutta, Jackson, and Le Breton's candidate stability theorem for the case in which candidates don't vote.

For any profile of linear voter preferences, a voting rule determines a winning candidate from any subset of candidates drawn from a fixed list of potential candidates based on the preferences of the voters over the candidates running for office. ${ }^{1}$ When there is no overlap between the candidates and voters, Dutta, Jackson, and Le Breton restrict attention to voting rules that respect unanimity in the sense that a candidate who is ranked first among the candidates on the ballot by all of the voters is elected. Their candidate stability theorem for the no overlap case shows that unanimity and candidate stability jointly imply that a single voter determines the outcome in any election in which all or all but one of the potential candidates is on the ballot. ${ }^{2}$

Dutta, Jackson, and Le Breton do not prove their theorem for the no overlap case directly; they instead show that it is a corollary to a more general impossibility theorem that allows for candidates who vote, but uses stronger candidate stability and unanimity conditions. When there is no overlap between the candidates and voters, their stronger candidate stability and unanimity conditions are equivalent to the conditions described above, at least when candidates rank themselves first, which is what Dutta, Jackson, and Le Breton assume. The proof of this strong candidate stability theorem is necessarily long and complicated.

\footnotetext{
${ }^{1}$ Rodríguez-Àlvarez [4] has considered the implications of candidate stability for multivalued voting procedures.

${ }^{2}$ When candidates are permitted to vote, Dutta, Jackson, and Le Breton have shown that candidate stability is incompatible with a weak unanimity condition that is consistent with a candidate ranking him- or herself first and a monotonicity condition that is satisfied by many common voting procedures.
} 
Although, as Dutta, Jackson, and Le Breton [2, p. 11] note, 'the logic of Arrow's theorem cannot be directly applied' to prove their strong candidate stability theorem, they nevertheless are able to use Wilson's [5] generalization of Arrow's Theorem at a key step in their argument. The proof provided here of Dutta, Jackson, and Le Breton's candidate stability theorem for the case in which candidates don't vote demonstrates that the incompatibility of their axioms follows fairly directly from a restricted-domain version of Arrow's impossibility theorem when there is no overlap between candidates and voters.

In Sections 2 and 3, respectively, I present Grether and Plott's Theorem and Dutta, Jackson, and Le Breton's candidate stability theorem for the case in which candidates and voters have no one in common. Section 4 is devoted to my proof of the latter result.

\section{THE GRETHER-PLOTT THEOREM}

Let $N=\{1, \ldots, n\}$ with $n \geq 2$ be a finite set of individuals and $X=$ $\left\{x_{1}, \ldots, x_{m}\right\}$ with $m \geq 3$ be a finite set of alternatives. An agenda is a nonempty subset of $X$. An ordering of $X$ is a reflexive, complete, and transitive binary relation on $X$. The corresponding strict preference relation $P$ and indifference relation $I$ are defined as follows: For all $x, y \in X$, (a) $x P y \leftrightarrow x R y$ and $\neg(y R x)$ and (b) $x I y \leftrightarrow x R y$ and $y R x$. A linear ordering of $X$ is an antisymmetric ordering; i.e., an ordering for which no two distinct alternatives are indifferent. Let $\mathcal{R}$ denote the set of all orderings and $\mathcal{L}$ denote the set of all linear orderings of $X$.

Each individual $i \in N$ has a preference ordering $R_{i} \in \mathcal{R}$ of $X$. A preference profile $\mathbf{R}=\left(R_{1}, \ldots, R_{n}\right)$ is an $n$-tuple of individual preference orderings. Two preferences $R^{1}, R^{2} \in \mathcal{R}$ coincide on $A \subseteq X$ if for all $x, y \in A$, $x R^{1} y \leftrightarrow x R^{2} y$. Two profiles $\mathbf{R}^{1}, \mathbf{R}^{2} \in \mathcal{R}^{n}$ coincide on $A \subseteq X$ if $R_{i}^{1}$ and $R_{i}^{2}$ coincide on $A$ for all $i \in N$.

The set of admissible profiles and/or the set of admissible agendas may be restricted a priori. The preference domain is $\mathcal{D}$, a nonempty subset of $\mathcal{R}^{n}$. The agenda domain is $\mathcal{A}$, a collection of nonempty subsets of $X$.

A social choice correspondence $C: \mathcal{A} \times \mathcal{D} \rightarrow X$ is a mapping that assigns a nonempty subset of the agenda to each admissible agenda and admissible profile. The set $C(A, \mathbf{R})$ is the choice set. If for all $A \in \mathcal{A}$ and all $\mathbf{R} \in \mathcal{D}$, $C(A, \mathbf{R})$ contains a single alternative, then $C$ is a social choice function. In this case, I write $x$ instead of $\{x\}$ when $\{x\}$ is the choice set. 
In its choice-theoretic formulation, Arrow's [1] Theorem demonstrates that the four Arrow social choice correspondence axioms are incompatible when the preference domain is unrestricted (i.e., $\mathcal{D}=\mathcal{R}^{n}$ ) and every nonempty subset of $X$ is an admissible agenda. The Arrow axioms are Arrow's Choice Axiom, Independence of Infeasible Alternatives, Weak Pareto, and Nondictatorship.

Arrow's Choice Axiom places restrictions on how choices are made out of different agendas for a fixed preference profile.

Arrow's Choice Axiom. For all $A^{1}, A^{2} \in \mathcal{A}$ and all $\mathbf{R} \in \mathcal{D}$, if $A^{1} \subseteq A^{2}$ and $C\left(A^{2}, \mathbf{R}\right) \cap A^{1} \neq \emptyset$, then $C\left(A^{1}, \mathbf{R}\right)=C\left(A^{2}, \mathbf{R}\right) \cap A^{1}$.

Informally, for a given profile $\mathbf{R}$, if the agenda $A^{1}$ is a subset of the agenda $A^{2}$ and the choice sets for these two agendas have at least one alternative in common, then the choice set for the smaller agenda consists of that part of the choice set for the larger agenda that is contained in the smaller agenda.

Independence of Infeasible Alternatives requires the choice set to be independent of preferences over alternatives not in the agenda.

Independence of Infeasible Alternatives. For all $A \in \mathcal{A}$ and all $\mathbf{R}^{1}, \mathbf{R}^{2} \in$ $\mathcal{D}$, if $\mathbf{R}^{1}$ and $\mathbf{R}^{2}$ coincide on $A$, then $C\left(A, \mathbf{R}^{1}\right)=C\left(A, \mathbf{R}^{2}\right)$.

For all $A \in \mathcal{A}$ and all $\mathbf{R} \in \mathcal{D}$, the weak Pareto set is

$$
\mathcal{P}(A, \mathbf{R})=\left\{x \in A \mid \nexists y \in A \text { such that } y P_{i} x \text { for all } i \in N\right\} .
$$

The Weak Pareto axiom requires the choice set to be a subset of the weak Pareto set.

Weak Pareto. For all $A \in \mathcal{A}$ and all $\mathbf{R} \in \mathcal{D}, C(A, \mathbf{R}) \subseteq \mathcal{P}(A, \mathbf{R})$.

For a nonempty set $A \subseteq X$ and an ordering $R \in \mathcal{R}$, the set of best alternatives in $A$ according to $R$ is

$$
B(A, R)=\{x \in A \mid x R y \text { for all } y \in A\} .
$$

An individual $d \in N$ is a dictator for the social choice correspondence $C: \mathcal{A} \times$ $\mathcal{D} \rightarrow X$ if $C(A, \mathbf{R}) \subseteq B\left(A, R_{d}\right)$ for all $A \in \mathcal{A}$ and all $\mathbf{R} \in \mathcal{D}$. That is, $d$ is a dictator if the choice set is always a subset of $d$ 's best alternatives in the agenda. Nondictatorship is the requirement that there be no dictator. 
Nondictatorship. There is no dictator.

Grether and Plott [3] investigated the consistency of the Arrow axioms when the only admissible agendas are those subsets of $X$ containing at least $k$ alternatives, where $k<|X|$.

$k$-Set Feasibility. There exists a positive integer $k<|X|$ such that $A \in \mathcal{A}$ if and only if $|A| \geq k$.

Grether and Plott assumed that the preference domain is unrestricted. Their theorem is also valid for the domain of linear preference profiles, and it is this version of their theorem that is relevant here.

Unrestricted Linear Preference Domain. $\mathcal{D}=\mathcal{L}^{n}$.

The Grether-Plott Theorem shows that the Arrow axioms are inconsistent with an unrestricted linear preference domain when the agenda domain satisfies $k$-Set Feasibility.

TheOREM 1. (Grether-Plott [3]) There is no social choice correspondence with an unrestricted linear preference domain that satisfies $k$-Set Feasibility, Arrow's Choice Axiom, Independence of Infeasible Alternatives, Weak Pareto, and Nondictatorship. ${ }^{3}$

\section{THE DUTTA-JACKSON-LE BRETON THEOREM}

The framework introduced in the previous section needs to be modified slightly in order to describe the Dutta-Jackson-Le Breton [2] model of strategic candidacy. The set $N$ is now interpreted as being the set of voters and the set $X$ is the set of potential candidates. As above, it is assumed that $|N| \geq 2$ and $|X| \geq 3$. I restrict attention to Dutta, Jackson, and Le Breton's impossibility theorem for the case in which no voter is a candidate. It is sometimes convenient to index the candidates using the set $M=\{1, \ldots, m\}$.

\footnotetext{
${ }^{3}$ Theorem 1 is established by showing that if it is possible to satisfy all of the Arrow axioms on the domain specified in the theorem, then it is possible to construct a social choice correspondence satisfying these axioms on the domain used in Arrow's Theorem, which is impossible. Because Arrow's Theorem is valid when the preference domain is $\mathcal{L}^{n}$, so is the Grether-Plott Theorem.
} 
Both voters and candidates have preferences over candidates. As in Section 2 , let $\mathbf{R}$ denote a profile of voters' preferences. A profile of candidates' preferences is a vector $\mathbf{R}_{c}=\left\{R_{n+1}, \ldots, R_{n+m}\right\}$, where $R_{n+j}$ is the preference ordering of the $j$ th candidate. Each voter can have any preference in $\mathcal{L}$. Each candidate also has a linear preference on $X$, but always prefers him- or herself to any of the other candidates. Let

$$
\mathcal{L}_{c}=\left\{\mathbf{R}_{c} \in \mathcal{L}^{m} \mid B\left(X, R_{n+j}\right)=\left\{x_{j}\right\} \text { for all } j \in M\right\} .
$$

Thus, the set of admissible profiles of preferences is $\mathcal{L}^{n} \times \mathcal{L}_{c}$.

Any subset of the set of potential candidates may stand for election. Let $\mathcal{X}$ denote the set of all nonempty subsets of $X$. A voting function is a social choice function $V: \mathcal{X} \times \mathcal{L}^{n} \times \mathcal{L}_{c} \rightarrow X$.

The outcome of an election only depends on the voters' preferences.

Independence of Nonvoters' Preferences. For all $A \in \mathcal{X}$, all $\mathbf{R} \in \mathcal{L}^{n}$, and all $\mathbf{R}_{c}^{1}, \mathbf{R}_{c}^{2} \in \mathcal{L}_{c}, V\left(A,\left(\mathbf{R}, \mathbf{R}_{c}^{1}\right)\right)=V\left(A,\left(\mathbf{R}, \mathbf{R}_{c}^{2}\right)\right)$.

A voting function satisfies Unanimity if candidate $x$ is chosen when all of the voters agree that $x$ is the best candidate running for office.

Unanimity. For all $A \in \mathcal{X}$ and all $\left(\mathbf{R}, \mathbf{R}_{c}\right) \in \mathcal{L}^{n} \times \mathcal{L}_{c}$, if $B\left(A, R_{i}\right)=\{x\}$ for all $i \in N$, then $V\left(A,\left(\mathbf{R}, \mathbf{R}_{c}\right)\right)=x$.

A voting function is Candidate Stable if each candidate prefers the outcome when all candidates are on the ballot to the outcome that would obtain if he or she withdrew from the election.

Candidate Stability. For all $j \in M$ and all $\left(\mathbf{R}, \mathbf{R}_{c}\right) \in \mathcal{L}^{n} \times \mathcal{L}_{c}, V\left(X,\left(\mathbf{R}, \mathbf{R}_{c}\right)\right)$ $R_{n+j} V\left(X \backslash\left\{x_{j}\right\},\left(\mathbf{R}, \mathbf{R}_{c}\right)\right)$.

Dutta, Jackson, and Le Breton use a strengthened version of the Nondictatorship axiom introduced in the preceding section. A voter $d \in N$ is a dictator for large elections for the voting function $V: \mathcal{X} \times \mathcal{L}^{n} \times \mathcal{L}_{c} \rightarrow X$ if $V\left(A,\left(\mathbf{R}, \mathbf{R}_{c}\right)\right)=x$ for all $A \in \mathcal{X}$ with $|A| \geq|X|-1$ and all $\left(\mathbf{R}, \mathbf{R}_{c}\right) \in \mathcal{L}^{n} \times \mathcal{L}_{c}$ for which $B\left(A, R_{d}\right)=\{x\}$. Informally, an individual is a dictator for large elections if this individual's most-preferred candidate is elected when at least $|X|-1$ candidates run for office.

Strong Nondictatorship. There is no dictator for large elections. 
Dutta, Jackson, and Le Breton also require the voting function to satisfy Independence of Infeasible Alternatives, modified in the obvious way to apply to preference profiles in $\mathcal{L}^{n} \times \mathcal{L}_{c}$. In this context, Independence of Infeasible Alternatives requires the election outcome only to depend on the preferences over candidates who enter the election.

Theorem 2 is the Dutta-Jackson-Le Breton candidate stability theorem for the case in which there is no overlap between candidates and voters.

Theorem 2. (Dutta-Jackson-Le Breton [2]) There is no voting function that satisfies Independence of Nonvoters' Preferences, Independence of Infeasible Alternatives, Unanimity, Candidate Stability, and Strong Nondictatorship.

Dutta, Jackson, and Le Breton also consider a stronger candidate stability axiom that requires the election outcome to be unaffected if a candidate withdraws who would lose if every candidate stood for office.

Strong Candidate Stability. For all $j \in M$ and all $\left(\mathbf{R}, \mathbf{R}_{c}\right) \in \mathcal{L}^{n} \times \mathcal{L}_{c}$, if $V\left(X,\left(\mathbf{R}, \mathbf{R}_{c}\right)\right) \neq x_{j}$, then $V\left(X,\left(\mathbf{R}, \mathbf{R}_{c}\right)\right)=V\left(X \backslash\left\{x_{j}\right\},\left(\mathbf{R}, \mathbf{R}_{c}\right)\right)$.

This axiom is a strengthening of Candidate Stability if the set of voters and candidates overlap. However, when no voter is a candidate, as is the case here, these conditions are equivalent for a voting function that satisfies Independence of Nonvoters' Preferences.

Lemma. (Dutta-Jackson-Le Breton [2]) If a voting function satisfies Independence of Nonvoters' Preferences, then it satisfies Candidate Stability if and only if it satisfies Strong Candidate Stability. ${ }^{4}$

If a voting function $V$ satisfies Independence of Nonvoters' Preferences, one can identify $V$ with a social choice function $C_{v}$ with domain $\mathcal{X} \times \mathcal{L}^{n}$ by setting, for all $A \in \mathcal{X}$ and all $\mathbf{R} \in \mathcal{L}^{n}$,

$$
C_{v}(A, \mathbf{R})=V\left(\left(A,\left(\mathbf{R}, \mathbf{R}_{c}\right)\right),\right.
$$

\footnotetext{
${ }^{4}$ I have only stated the part of Lemma 2 in Dutta, Jackson, and Le Breton [2] that deals with Candidate Stability. Their lemma also assumes that the voting function satisfies Independence of Infeasible Alternatives and Unanimity, but these assumptions are not needed to show the equivalence of Candidate Stability and Strong Candidate Stability.
} 
for any $\mathbf{R}_{c} \in \mathcal{L}_{c}$. The Candidate Stability axiom only makes sense if the candidates' preferences are included in the domain, and so it can't be stated directly in terms of a social choice function. However, Strong Candidate Stability can be easily reformulated as an axiom for a social choice function.

\section{A PROOF OF THEOREM 2}

In this section, the social choice function $C_{v}$ is used in combination with the Grether-Plott Theorem to prove Theorem 2.

Step 1. Supppose that the voting function $V: \mathcal{X} \times \mathcal{L}^{n} \times \mathcal{L}_{c} \rightarrow X$ satisfies all the assumptions of Theorem 2 except Strong Nondictatorship. Let $C_{v}: \mathcal{X} \times$ $\mathcal{L}^{n} \rightarrow X$ be the social choice function corresponding to $V$ defined in the preceding section. Let $C: \mathcal{A}^{m-1} \times \mathcal{L}^{n} \rightarrow X$ be the restriction of $C_{v}$ to $\mathcal{A}^{m-1} \times$ $\mathcal{L}^{n}$, where $\mathcal{A}^{m-1}=\{A \in \mathcal{X}|| A \mid \geq m-1\}$.

Because $V$ satisfies Independence of Infeasible Alternatives and Unanimity, so does $C$ (with the definition of Unanimity modified in the obvious way to apply to the domain $\mathcal{A}^{m-1} \times \mathcal{L}^{n}$ ). By the Lemma, $V$ satisfies Strong Candidate Stability. Because $C$ is a function and $V$ satisfies Independence of Nonvoters' Preferences, the satisfaction of Strong Candidate Stability by $V$ is equivalent to the satisfaction of Arrow's Choice Axiom by $C$.

Step 2. Next, I show that for any $\mathbf{R} \in \mathcal{L}^{n}, C(X, \mathbf{R})$ is contained in the weak Pareto set. On the contrary, suppose that there exists an $\mathbf{R} \in \mathcal{L}^{n}$ and $x, y \in X$ such that $x P_{i} y$ for all $i \in N$, but $C(X, \mathbf{R})=y$. Without loss of generality, suppose that $x=x_{1}$ and $y=x_{2}$.

By Arrow's Choice Axiom, $C\left(X \backslash\left\{x_{3}\right\}, \mathbf{R}\right)=x_{2}$. Consider a profile $\mathbf{R}^{1} \in \mathcal{L}^{n}$ that coincides with $\mathbf{R}$ on $X \backslash\left\{x_{3}\right\}$ and has $x_{3}$ ranked last by all $i \in N$. By Independence of Infeasible Alternatives, $C\left(X \backslash\left\{x_{3}\right\}, \mathbf{R}^{1}\right)=x_{2}$. It then follows from Arrow's Choice Axiom that $C\left(X, \mathbf{R}^{1}\right) \subseteq\left\{x_{2}, x_{3}\right\}$.

Arrow's Choice Axiom now implies that $C\left(X \backslash\left\{x_{4}\right\}, \mathbf{R}^{1}\right) \subseteq\left\{x_{2}, x_{3}\right\}$. Consider a profile $\mathbf{R}^{2} \in \mathcal{L}^{n}$ that coincides with $\mathbf{R}^{1}$ on $X \backslash\left\{x_{4}\right\}$ and has $x_{4}$ ranked last by all $i \in N$. By Independence of Infeasible Alternatives, $C\left(X \backslash\left\{x_{4}\right\}, \mathbf{R}^{2}\right)=C\left(X \backslash\left\{x_{4}\right\}, \mathbf{R}^{1}\right) \subseteq\left\{x_{2}, x_{3}\right\}$. Hence, by Arrow's Choice Axiom, $C\left(X, \mathbf{R}^{2}\right) \subseteq\left\{x_{2}, x_{3}, x_{4}\right\}$.

Repeated use of this argument leads to the conclusion that $C\left(X, \mathbf{R}^{m-2}\right) \subseteq$ $\left\{x_{2}, \ldots, x_{m}\right\}$ for some profile $\mathbf{R}^{m-2} \in \mathcal{L}^{n}$ that coincides with $\mathbf{R}$ on $\left\{x_{1}, x_{2}\right\}$ and has $x_{1} P_{i}^{m-2} x_{3}, x_{2} P_{i}^{m-2} x_{3}$, and $x_{3} P_{i}^{m-2} x_{4} P_{i}^{m-2} \ldots P_{i}^{m-2} x_{m}$ for all $i \in N$. Because $x_{1} P_{i} x_{2}$ for all $i \in N$, we therefore have $x_{1} P_{i}^{m-2} x_{j}$ for all $i \in N$ and all $j=2, \ldots, m$. Hence, Unanimity is violated. 
Step 3. I now show that for all $x \in X$ and all $\mathbf{R} \in \mathcal{L}^{n}, C(X \backslash\{x\}, \mathbf{R})$ is contained in the weak Pareto set. On the contrary, suppose that there exist distinct $x, y, z \in X$ and $\mathbf{R} \in \mathcal{L}^{n}$ such that $y P_{i} z$ for all $i \in N$, but $C(X \backslash\{x\}, \mathbf{R})=z$. Consider a profile $\mathbf{R}^{1} \in \mathcal{L}^{n}$ that coincides with $\mathbf{R}$ on $X \backslash\{x\}$ and has $x$ ranked last by all $i \in N$. By Independence of Infeasible Alternatives, $C\left(X \backslash\{x\}, \mathbf{R}^{1}\right)=z$. By Arrow's Choice Axiom, $C\left(X, \mathbf{R}^{1}\right) \subseteq$

$\{x, z\}$, which contradicts what was established in Step 2 because $y$ Pareto dominates both $x$ and $z$ in $\mathbf{R}^{1}$.

Step 4. By Step 1, $C$ satisfies Arrow's Choice Axiom and Independence of Infeasible Alternatives. Steps 2 and 3 show that $C$ satisfies Weak Pareto. $C$ satisfies $k$-Set Feasibility with $k=m-1$. Therefore, by the Grether-Plott Theorem (Theorem 1), $C$ is dictatorial. But $C$ being dictatorial is equivalent to $V$ having a dictator for large elections. Thus, $V$ does not satisfy Strong Nondictatorship, which completes the proof of Theorem 2.

\section{REFERENCES}

1. K. J. Arrow (1963), Social Choice and Individual Values, Second Edition, John Wiley and Sons, New York.

2. B. Dutta, M. O. Jackson, and M. Le Breton, Strategic candidacy and voting procedures, Econometrica, forthcoming.

3. D. M. Grether and C. R. Plott (1982), Nonbinary social choice: An impossibility result, Review of Economic Studies 49, 143-149.

4. C. Rodríguez-Àlvarez (2001), Candidate stability and voting procedures, unpublished manuscript, Departament d'Economia i Història Econòmica, Universitat Autònoma de Barcelona.

5. R. B. Wilson (1972), Social choice without the Pareto principle, Journal of Economic Theory 5, 478-486. 